Received Date: 02/06/2016

Accepted Date: 03/01/2017

Article Type: Main Research Article

\title{
A mobile health intervention promoting healthy gestational weight gain for women entering pregnancy at a high body mass index: the txt4two pilot randomised controlled trial
}

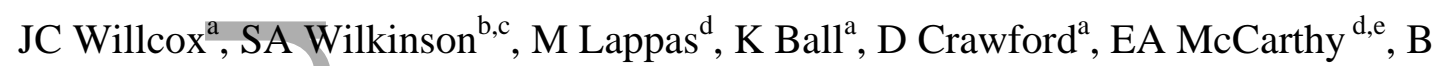
Fjeldsoe $^{\mathrm{f}}$, R Whittaker ${ }^{\mathrm{g}}, \mathrm{R}^{\mathrm{R}}$ Maddison $^{\mathrm{a}}$, KJ Campbell ${ }^{\mathrm{a}}$

${ }^{a}$ Institute for Physical Activity and Nutrition, Deakin University, Victoria, Australia

${ }^{\mathrm{b}}$ Mater Research Institute- University of Queensland, South Brisbane, Queensland, Australia.

${ }^{\mathrm{c}}$ Department of Nutrition \& Dietetics, Mater Mothers Hospital, South Brisbane, Queensland, Australia

${ }^{\mathrm{d}}$ Department of Obstetrics and Gynaecology, University of Melbourne, Victoria, Australia

${ }^{\mathrm{e}}$ Mercy Hospital for Women, Melbourne, Victoria, Australia

${ }^{\mathrm{f}}$ School of Public Health, The University of Queensland, Herston, Queensland, Australia

g National Institute for Health Innovation, University of Auckland, Auckland, New Zealand Correspondance:

JC Willcox

M Institute for Physical Activity and Nutrition, Deakin University, 221 Burwood Highway, Burwood, Victoria, 3125, Australia

Ejwillcox@deakin.edu.au

$\mathrm{T}+61392468733$

This is the author manuscript accepted for publication and has undergone full peer review but has not been through the copyediting, typesetting, pagination and proofreading process, which may lead to differences between this version and the Version of Record. Please cite this article as doi: $10.1111 / 1471-0528.14552$

This article is protected by copyright. All rights reserved 


\section{Short title}

mHealth RCT to optimise GWG for high BMI women

Abstract

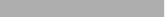<smiles>[O]</smiles>

Objective To determine the feasibility and effectiveness of an mHealth intervention promoting healthy diet, physical activity and gestational weight gain in pregnant women.

Design Randomised controlled trial (RCT)

Setting Australian tertiary obstetric hospital

Population One hundred pregnant women who were overweight or obese prior to pregnancy.

Methods Women recruited at the first antenatal clinic visit were randomised to either an intervention or a control group. The intervention consisted of a tailored suite of strategies delivered (from first antenatal visit until 36 weeks gestation) via multiple modalities available on mobile devices.

Outcomes The primary outcome was intervention feasibility and secondary outcomes were objectively-measured changes in gestational weight gain (GWG) and self-reported dietary intake and physical activity.

Results Ninety one women completed the study. Delivery to protocol provides evidence of program feasibility. Most women engaged regularly with the program with the majority (97.6\%) reporting that the intervention was helpful. Secondary outcomes demonstrated a significantly lower GWG in the intervention group $(7.8 \mathrm{~kg} \pm 4.7$ versus $9.7 \mathrm{~kg} \pm 3.9 ; \mathrm{p}=$ 0.041) compared to the control group at intervention completion. Intervention group women reported significantly smaller reductions in total, light- and moderate-intensity physical activity from baseline to completion of the intervention $(\mathrm{p}=0.001)$ compared with the control group, but no differences in consumption frequencies of key food groups. 
Conclusion An intervention that aimed to deliver healthy diet, physical activity and GWG guidance utilising innovative technology can be feasibly implemented and produce positive physical activity and GWG outcomes.

Key words: Pregnancy, mHealth, diet, physical activity, gestational weight gain, intervention

Trial registration: Australian New Zealand Clinical Trials Registry: ACTRN: 12614000288628.

https://www.anzctr.org.au/Trial/Registration/TrialReview.aspx?id=365956\&isReview=true Date of registration: 19 March 2014

Tweetable abstract: txt4two mHealth study improved gestational weight gain and physical activity in pregnant women with high BMIs

\section{Introduction}

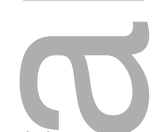

Animal and human studies suggest various mechanisms by which suboptimal maternal lifestyle confers health risks for offspring and potentially grandchildren. ${ }^{1,2}$ Putative mechanisms include epigenetic histone methylation changes related to diet quality, ${ }^{1}$ or a mismatched epigenetic pathway, cued by prenatal undernutrition in which the foetus forecasts an adverse future environment and changes its developmental trajectory accordingly. ${ }^{2}$

Gestational weight gain (GWG) within recommendations is associated with positive health outcomes for both mother and child..$^{3-6}$ An estimated 35 to $65 \%$ of women in developed countries exceed the internationally recognised Institute of Medicine (IOM) GWG guidelines. ${ }^{7-10}$ Women who are overweight or obese prior to pregnancy are at a three-fold increased risk of exceeding GWG guidelines compared with those who are not. ${ }^{11}$

Interventions and meta-analyses of trials promoting healthy GWG are growing in number and scope $^{12}$. The recent Cochrane meta-analysis of interventions to promote healthy GWG reported that those involving diet, exercise, or both, reduced the risk of excess GWG on average by $20 \%$ (Relative risk $0.80,95 \%$ CI $0.73,0.87$ ). ${ }^{12}$ A limitation of many previous interventions has been the heavy reliance on intensive support from clinical providers. The high costs associated with intensive interventions are known to limit reach and scalability, 
and to increase disparities in service provision. ${ }^{13}$ Further, traditional approaches with regular group or face-to-face health professional visits may not be convenient for many women. ${ }^{14}$

Interventions delivered via electronic mobile devices, commonly referred to as mHealth, ${ }^{15}$ offer an opportunity to provide trusted source information across demographic groups, incorporating behavioural change practices through a low-cost, easy access method. ${ }^{16,17}$ Research on mHealth interventions to promote healthy GWG is limited and there has been a call to build the evidence base with health behaviour change support in real-life settings. ${ }^{17,18}$ Evidence to date is largely informed by three pilot studies, two RCTs and one feasibility study, of text message based interventions to promote healthy GWG. ${ }^{19-21}$ These pilot studies have demonstrated feasibility and limited efficacy, but contain limitations such as small sample sizes, marked attrition ${ }^{19,20}$, recruitment bias ${ }^{19}$ and additional health personnel intervention $^{21}$. There remain opportunities to pilot intervention models promoting healthy diet, physical activity and GWG that are primarily delivered by mobile devices.

The aim of this pilot study was to test the feasibility and effectiveness of an mHealth intervention (txt4two) to promote healthy diet, physical activity and weight gain in pregnant women who were overweight or obese prior to pregnancy. This paper reports on the primary outcome of feasibility of intervention implementation and the secondary outcomes of GWG, dietary intake and physical activity changes.

\section{Methods}

The study was a randomised controlled trial (RCT) comparing the txt4two mHealth intervention, with usual care in pregnant women who were overweight or obese prior to pregnancy. The methodology for this paper has been previously published and is briefly outlined below.$^{22}$ The study aimed to promote healthy diet, physical activity and GWG within the IOM guidelines using multi mHealth modalities. The mHealth Development and Evaluation framework informed the development of the study and the preceding formative work. $^{23,24}$ The study was guided by the SPIRIT 2013 statement ${ }^{25}$ and the CONSORT eHEALTH guidelines. ${ }^{26,27}$

\section{Participants and recruitment}


Eligible women were identified at their first hospital antenatal visit to a university-affiliated maternity hospital in Melbourne, Australia. Eligibility criteria included women with a singleton, live gestation between $10^{+0}-17^{+6}$ weeks; self-reported pre-pregnancy BMI $>25 \mathrm{~kg} / \mathrm{m}^{2}$; able to speak, read and write English; and owned a mobile phone. Exclusion criteria included: < 18 years of age; multiple pregnancy; comorbidities requiring significant medical and/or dietary management; or discontinuation of hospital care. Following medical record screening, the research assistant outlined the study and provided a plain language statement for informed consent.

\section{Sample size and randomisation}

The primary outcome of this study was the assessment of feasibility. This informed the sample size of 100 women which was considered sufficient to allow for the estimation of the standard deviation of GWG, a continuous variable, and to provide reliable data on recruitment and intervention delivery parameters required for planning of a larger intervention trial. $^{28}$

Randomisation utilised computer-generated random numbers. Numbered cards allocating women to either the intervention or control groups were placed in opaque, sequentially numbered envelopes. Given the nature of the intervention participants could not be blinded to group assignment.

\section{Intervention}

The txt4two intervention was an mHealth intervention which aimed to promote healthy diet, physical activity and GWG from recruitment to 36 weeks gestation. The intervention content was developed according to evidence-based guidelines including the IOM guidelines for $\mathrm{GWG}^{10}$, the Australian Dietary Guidelines for pregnancy ${ }^{29}$ and the Australian Physical Activity \& Sedentary Behaviour Guidelines for Adults ${ }^{30}$.The IOM guidelines for GWG were promoted with encouragement to stay within the guidelines. Information and guidance to professional help was provided for excess or insufficient weight gain. The Australian Dietary Guidelines emphasise the replacement of sugar sweetened beverages, increased fruit and vegetable intake, reduction of discretionary food groups and consumption of regular meals. 
The physical activity guidelines emphasise 30 minutes of moderate intensity physical activity on most, if not all, days of the week, reduction of sedentary behaviour. Behaviour change guidance was informed by Social Cognitive Theory ${ }^{31}$ and the CALO-RE taxonomy of behaviour change techniques. ${ }^{32}$ The taxonomy of behaviour change techniques allows a rigorous and precise method of characterising the active content of interventions.

The multi-modality delivered intervention included tailored text messages, access to a responsive information website viewable on mobile devices, video messages, and chat room interaction via Facebook ${ }^{\circledR}$ (Menlo Park, CA, USA). (Described in Supplementary tables S1 and S2). With the exception of the initial interview and booklet (described below), the intervention was completely accessible on mobile devices. The text messages were administered yia University of Queensland's CPRC SMS Software. Consistent with behaviour change research, the intervention components were mapped to behaviour change techniques described by Michie and colleague's ${ }^{32}$. Specific techniques were informed by Social Cognitive Theory and included goal setting, self-monitoring and review of goals and outcomes. ${ }^{22}$ (Described in Supplementary table S2).

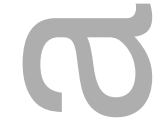

At baseline the trained researcher conducted the initial interview where they outlined the intervention and provided a booklet outlining the txt4two program, weight tracking and goal setting. In addition, the researcher discussed appropriate GWG targets, individual GWG monitoring and recording, and asked the woman to set a nutrition or physical activity goal to work towards the above-mentioned evidence-based recommendations.

Following the initial interview, intervention participants received four to five individuallytailored, interactive text messages per week, a frequency found acceptable in other mHealth interventions. . $^{33,34}$ The texts delivered information specific to the individual's gestational week, encouragement of positive health behaviours, monitoring of individual goals and encouragement of self-monitoring of GWG. Texts were developed and mapped according to the behaviour change techniques by JW and BF. Women chose the frequency of texts that aimed to: prompt review of their weight (weekly or fortnightly); and check their behavioural goals (weekly or fortnightly). Confirmation of text message receipt was assessed by telephone early in the intervention. 
A study specific website outlined intervention content information (txt4two website archived by WebCite ${ }^{\circledR}$ at http://www.webcitation.org/6QR3k6uaM). Short videos featuring an obstetrician, dietitian or physiotherapist were embedded in the website and outlined the benefits of the intervention and explained the components. The private Facebook $₫$ chat page, only accessible to txt4two participants, encouraged interaction with other participants and posing of questions to health professionals. The website and Facebook group were promoted and linked in the text message content.

\section{Standard care}

Participants in both intervention and standard care groups were mailed brief information brochures containing advice regarding diet and physical activity prior to the first hospital visit and were also encouraged to weigh at first visit. This information and advice are part of usual maternity care for women at the study hospital.

\section{Data collection and outcome measures}

\section{Primary outcome}

Intervention feasibility was measured by program-generated data and participant-reported data. Feasibility, including a process evaluation data, was informed by the Process-Evaluation Plan for Assessing Health Programme Implementation ${ }^{35}$ and the eCONSORT guidelines ${ }^{26}$. An outline of the process evaluation may be found in Table S3: Process Evaluation. Indicators of feasibility included:

1. Recruitment of participants (numbers screened, approached and recruited) and retention (numbers completing trial)

2. Intervention protocol delivery and fidelity (delivery of intervention content described in protocol, external validity with additional services consulted e.g. dietitian, gestational diabetes education)

3. Dose delivered (contacts delivered); intervention engagement (contact with the program elements (text program, website and Facebook analytics) and self-reported use of program elements),

4. Acceptability (self-reported Likert scales judging acceptability of program and elements).

\section{Secondary outcomes}


The secondary outcomes of GWG, diet and physical activity were assessed at baseline and 36 week visit according to the following measurements:

1. Weight was measured by trained staff with Wedderburn WM301 scales with $0.1 \mathrm{~kg}$ accuracy, in light clothing without shoes. Height was measured on a calibrated stadiometer. GWG (intervention) was defined as the difference in weight between baseline and 36 week visit. GWG (pregnancy) was defined as the difference between participant's estimated pre-pregnancy weight and 36 week visit weight. The proportion of women whose GWG (pregnancy) exceeded the IOM recommendations based on pre-pregnancy BMI (ppBMI) was calculated. 2. Diet assessed by frequency of key food group consumption by a published food frequency questionnaire ${ }^{36}$ and included: fruit, vegetables, non-core sweet and savoury foods, non-core drinks, lean protein, dairy, water and alcohol. The questionnaire was based on several previously published and validated Australian nutrition surveys ${ }^{37-39}$ and has shown good convergent validity among women of childbearing age. ${ }^{40}$

3. Physical activity level was assessed by the previously validated Pregnancy Physical Activity Questionnaire (PPAQ) ${ }^{41,42}$ including time spent in sedentary, light, moderate and vigorous activity.

\section{Compensation}

To promote retention, participants were provided with a \$20 shopping voucher on completion of the evaluation at baseline and 36 weeks. In addition, intervention participants received a \$20 iTunes voucher to compensate for data usage incurred by involvement in the txt4two program.

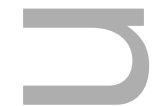

\section{Statistical analysis}

Consistent with revised Consolidated Standards of Reporting Trials guidelines ${ }^{43}$ analyses included only those participants who had completed the study $(n=91)$. Analyses were conducted using Stata (Release 13; StataCorp, College Station, TX, USA). The participant group allocation was re-coded by an independent researcher to ensure that the data analyst 
was blinded to allocation. Feasibility and participant characteristics were analysed with categorical variables reported as numbers and percentages and continuous variables as means and standard deviations.

Linear regression models were used to examine intervention effects on GWG, dietary and physical activity outcomes. All models controlled for the baseline value of the respective outcome and for a priori-determined covariates: age, education (for socioeconomic status), pre-pregnancy BMI (for IOM GWG recommendations), parity (for additional education in previous pregnancies), gestational age (for GWG differential) gestational diabetes (for additional education) and number of days in the study (for intervention dosage). Seeing a dietitian (for additional education) was excluded as a covariate because of small numbers of participants $(n=4)$ reporting this.

\section{Results}

\section{Primary outcome - Feasibility}

\section{Recruitment and retention}

As shown in Figure 1, 789 women were screened between 29/05/2014 and 10/10/2014, 689 women were excluded because they did not meet eligibility criteria $(n=617)$ or declined involvement $(n=19)$. One hundred women provided consent and were randomised to the intervention or control arms. Four women from each arm withdrew early in the intervention due to miscarriage or pregnancy complications and one woman withdrew from the intervention arm citing dislike of the intervention.

Baseline sociodemographic characteristics of the 91 women included in the analyses are presented in Table 1. Mean age of participants was 32.5 years with a mean gestation of 15.5 weeks at recruitment. Nearly one half of women (46\%) were nulliparous. Approximately one quarter of women were born overseas. The mean self-reported ppBMI was $31.0 \mathrm{~kg} / \mathrm{m}^{2}$.

Participants who did not complete the study $(n=9)$ differed only in baseline characteristics being recruited earlier in pregnancy than those who completed $(13.6 \pm 2.4$ weeks compared with $15.5 \pm 2.0$ weeks; $\mathrm{p}=0.008$ ). Weight data was available for all completing participants at baseline and 36 weeks. Self-reported diet and physical activity data at 36 weeks was obtained 
for 45 of 46 control women and 44 of 45 intervention women. Self-reported evaluation of the txt4two intervention was obtained from 43 of 45 intervention women.

\section{Fidelity}

Program generated data indicated that the intervention was implemented to protocol ${ }^{22}$ with the exception of two events. Two women received texts out of sequence, due to human error, which was detected and remedied in the first week of the intervention. Assessment of contamination revealed the onset of gestational diabetes mellitus in 11 of control and six intervention group women, resulting in attendance for all these women at a one off group education session with a dietitian and diabetes nurse educator. Independent of the intervention or gestational diabetes mellitus treatment, one control and three intervention participants consulted a dietitian on one occasion each.

\section{Dose and engagement}

The average duration of participation in the study was 145 days (standard deviation (SD) 15). The initial interview on average lasted 10 minutes. During the interview, 35 women (78\%) chose to set physical activity, rather than dietary, goals as their focus behaviour. Forty percent of women $(n=18)$ chose to receive a goal review text weekly and $38 \%(n=17)$ a weight review text weekly. The remainder received these texts every two weeks along with three weekly gestational tracking and dietary and physical activity behaviour texts. All women reported reading the booklet, with $62 \%$ of women reading it for 30 minutes or more while the remainder read for less than 30 minutes. At 36 weeks, the majority of participants (40/42) reported setting regular behaviour change goals throughout the intervention. Self weighing frequency varied with $24(57 \%)$ women recounting weighing once or twice per month, $13(31 \%)$ once per week and $5(12 \%)$ a few times per week or every day. Twenty participants $(48 \%)$ described recording their weight on the weight chart every one or two weeks, $7(17 \%)$ not at all and the remainder once a month or every few months.

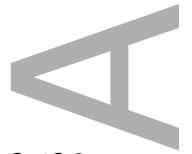

Overall 3629 texts were delivered to the intervention women with a mean of 81 texts per participant or four to five per week. The majority of women (43/45: 96\% ) replied to the texts, specifically goal checks and weight checks, with 506 texts or a mean of 11 reply texts per participant being received (expected texts equals 684). All women described receiving 
regular texts and the majority reported reading "all" $(33 / 42 ; 79 \%)$ or "most" $(8 / 42: 19 \%)$ texts.

The txt 4 two research team displayed 50 posts on the private Facebook® page. Fourteen participants (31\%) joined the private Facebook® page. These women recorded 558 views, a mean of 40 per participant, submitted seven posts and two questions, and indicated 18 likes.

The website averaged 1258 hits per month (number of files downloaded on a site) and 39 unique visitors per month (individuals who arrive at the website and browse). This averaged a mean of 0.87 visits per participant per month. The most popular pages included food ideas, maintaining core strength and the role of nutrients in pregnancy, all of which were promoted by to the text messages. The ten videos posted on the website were viewed 153 times in total. The most frequently viewed videos were those that were promoted by the text messages (i.e. the txt4two introduction, the benefits of exercise, and the obstetrician discussing GWG). While the videos and website were intentionally coded so not to be searchable on search engines, they were not password protected and hence may have been accessed by those outside the study.

\section{Acceptability}

Intervention participants were asked their views of the txt4two intervention and its constituent elements. Perceived helpfulness of the intervention and behaviour change elements are presented in Supporting Information Table S4. The majority of participants $(97.6 \%)$ judged the intervention to be some degree of helpful for developing healthier habits in pregnancy with 23 (55\%) rating it "extremely" or "very" helpful. Of those who reported setting regular goals during the intervention (40/43), goal setting was found "extremely" or "very" helpful for 12 (30\%), "moderately" helpful for 21 (53\%) and a "little" helpful for 7 $(17 \%)$. For those that self-weighed, 23 (55\%) described the process as "extremely" or "very" helpful, $16(38 \%)$ "moderately" or a "little" helpful and $3(7 \%)$ as "not at all" helpful. For those that replied to the weight check texts $(n=41) 15(41 \%)$ reported it "extremely" or "very" helpful, 16 (43\%) "moderately" or a "little" helpful and $6(16 \%)$ "not at all" helpful. The timing of the texts was rated as acceptable by all and the number of text was found to be "just right" by $33(79 \%)$ and "too many" by $9(21 \%)$.

\section{Secondary outcomes}




\section{Impact on GWG, diet and physical activity}

The intervention effects on GWG, unadjusted and adjusted for key covariates are presented in supporting tables S5 and S6. Adjusted analysis showed there was a significant difference in GWG between groups, with intervention group participants gaining 7.8kg and control group $9.7 \mathrm{~kg}$ ( $\mathrm{p}=0.041$ ). The proportion of women exceeding the GWG (pregnancy) recommendations by the IOM did not differ significantly between the intervention (21/45) and control groups (28/46).

The impacts on physical activity and diet for the intervention period are presented in supporting table S5 Self-reported physical activity: between group comparisons of intervention effect. Women randomised to the intervention group were less likely to reduce their minutes of total daily physical activity over the course of the intervention compared with women receiving standard care $(\mathrm{p}=0.001)$. These findings were driven by significant differences in adjusted light $(\mathrm{p}=0.006)$ and moderate $(\mathrm{p}=0.005)$ physical activity. No significant differences between groups were seen with sedentary or vigorous activity at 36 weeks gestation.

Women randomised to the intervention group did not demonstrate any significant differences in self-reported frequency of consumption of any of the food groups in unadjusted or adjusted analyses, in comparison to the control group. Data is presented in supporting table S6 Selfreported diet: between group comparisons of intervention effect.

\section{Discussion

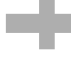

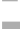

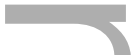 \\ Main findings}

This study has extended the design and results of previous pilot mHealth and pregnancy studies ${ }^{19-21}$ by demonstrating feasibility and acceptability of an mHealth intervention promoting healthy diet, physical activity and healthy GWG, within a public hospital outpatient clinic setting. It also showed statistically significant and meaningful reductions in GWG and maintenance of physical activity across pregnancy in the intervention group 
compared with the control group participants. This study did not demonstrate significant differences in diet or meeting of IOM GWG guidelines.

This was only the third RCT mHealth intervention study to investigate GWG modification. In comparison to the results of the two previous studies ${ }^{20,21}$, this RCT achieved significant GWG with greater participant numbers (91 versus 23-54), lower attrition (9\% versus 13 $30 \%)$ and limited face to face intervention. While the Herring and colleagues study $(n=54)$ demonstrated a $3.1 \mathrm{~kg}$ difference between groups (adjusted $\mathrm{p}=0.045$ ), the mHealth components (text messages and Facebook) augmented biweekly health coaching calls. ${ }^{21}$ In the Pollak and colleagues study $(n=23)$, the completing intervention participants exhibited a $2.7 \mathrm{~kg}$ difference in mean weight from the control group but was underpowered to demonstrate significance $(\mathrm{p}=0.24)^{20}$.

\section{Strengths and limitations}

The iterative development of this intervention utilising an mHealth Development and Evaluation framework ${ }^{44}$ which included formative research ${ }^{23}$, concept testing ${ }^{24}$ and a pilot study, was a strength of this study. Formative and conceptual investigation in the target group is an important first step in developing intervention approaches most likely to be feasible, appealing to, and effective in the target group. ${ }^{44,45}$ Another strength was the theory-based design with the intervention constructed specifically to target key behaviour change mediators from the behaviour change taxonomy. ${ }^{32}$ It is suggested that studies closely aligned with behaviour change theory and behaviour change techniques will be most effective in changing targeted health outcomes. ${ }^{46,47}$ The inclusion of multiple modalities or technological elements (e.g. combination of texts, website, videos and social media) to appeal to a range of preferences and learning styles was another strength. Multiple modalities for mHealth interventions was identified as being important by women in lead-up concept testing. ${ }^{24}$ The target audience's common utilisation of these modalities offers potential for sustainable provision of this intervention within existing models of antenatal care.

A limitation of this study was the potential for recall bias and socially desirable reporting associated with self-reported pre-pregnancy weight, diet and physical activity. This is a common concern for GWG, dietary and physical activity studies but given the purpose, size and budget of this study, more detailed assessments were not feasible. Food frequency questionnaires are a simplified form of the diet history and hence limit detection of dietary 
change when compared to more valid and reliable measures. ${ }^{48}$ This may have contributed to the lack of detected dietary change. A larger trial would require more valid and reliable measures of diet, for example use of multi pass three day dietary recall or food record. ${ }^{49}$ This study was conducted from a single large tertiary hospital, which may affect generalisability. Further, testing the feasibility of recruiting participants and delivering the initial interview by hospital clinic staff, rather than research staff, will be important in terms of scalability and generalisability in further trials.

\section{Interpretation}

The acceptability and engagement outcomes of this study were consistent with than those of other mHealth interventions with using similar process evaluation measures. ${ }^{19,20,50}$ For example in an eight week mHealth weight loss study with 53 adults in New Zealand, 66\% $(35 / 53)$ of participants reported reading "all" or "most" of the texts in comparison to $98 \%$ of participants in txt4two. Further, in that weight loss study, $42 \%$ of completer participants rated the program as "extremely helpful" or "very helpful" in comparison to $54 \%$ in the txt4two study. The behavioural aspects of both interventions, for example goal setting and monitoring, known to be key elements of behaviour change, were well received by participants.

The intervention demonstrated a significant intervention effect of meaningful magnitude. This effect is similar to outcomes from more intensive interventions described in the literature. $^{12,51}$ For example, a meta-analysis of 44 RCTs promoting healthy GWG $(n=7278)$ reported a significant reduction in intervention compared to control group GWG of $1.42 \mathrm{~kg}$ when all diet, physical activity and mixed approach interventions were analysed together. ${ }^{51}$ As such our results suggest potential effects similar to other programs delivered via face-toface, group and weight monitoring interventions. ${ }^{51}$ The mHealth approach may also offer economic advantages with mHealth interventions showing benefits from both health and cost saving perspectives. ${ }^{52}$ Economic analysis was not undertaken in this study and will be important to include in further trials.

While the txt4two program demonstrated a significant difference in GWG, it did not show a difference in the proportion of women exceeding GWG guidelines. This is consistent with a 
number of other studies. ${ }^{53,54}$ This may be in part due to insufficient power but also the late recruitment of women in pregnancy at 15.5 weeks. Further research is warranted to understand how to access women earlier in pregnancy and potentially preconception.

Research suggests that goal setting and self-monitoring may be among the most effective behaviour change techniques in promoting healthy GWG. ${ }^{46,55}$ In the txt4two intervention, women were encouraged to set a tailored behavioural goal (diet or physical activity) of their choice (which was then referred to throughout the text messages). Eighty percent of women chose to focus on a physical activity, rather than dietary, goal at intervention commencement. This may, in part, explain the significant differences seen for physical activity outcomes. The process of setting and monitoring physical activity goals which then translated into improvements in physical activity is proposed in Kim and colleagues study with 1116 US women pregnant and post-partum women. ${ }^{56}$ In that study, goal setting and self-monitoring helped to maintain positive intentions during pregnancy, with repeated self-monitoring transferring positive intentions into actual behaviour change.

Just $20 \%$ of women chose to set diet-related goals. This may have contributed to the null effect on diet. Evidence supports individual goal setting, with self-determined changes more likely to be enacted. ${ }^{31,32}$ Speculatively, the majority of women may have chosen a physical activity goal as firstly, it may have been easier to understand and set a goal with a single component behaviour, such as physical activity, rather than a multi-component dietary behaviour. Secondly, self-efficacy has been identified as a key determinant of enabling positive dietary and physical activity behaviours in pregnancy ${ }^{57}$ and women may have felt more confident to nominate physical activity changes. Moreover, pregnant women are more likely to nominate barriers than enablers to changing behaviour particularly diet changes which impacted others, such as family. Additionally previous research has shown the "minor disorders of pregnancy" such as food cravings, nausea and vomiting can be barriers to women making healthy dietary changes. ${ }^{58}$ Thirdly, research suggests that some women may view pregnancy as an opportunity for less dietary restraint than other times in the life course. ${ }^{59}$ Lastly, there is evidence that people tend to view their dietary patterns as better than others (optimistic bias) and so may have felt less need to set a dietary goal. ${ }^{60}$ Further research into the best ways to assist women in making diet behaviour change goals would be helpful in designing and implementing GWG interventions. 
The pilot nature of this study meant that short term or long term health outcomes were not assessed, although limitation of GWG related to the intervention is an encouraging finding. A larger study funded for appropriately detailed and longitudinal follow up would be required to identify if pregnancy complications are reduced and longer term maternal and family health improved when women engage in mHealth supported lifestyle change during pregnancy. Further, a larger trial could establish the efficacy of individual behaviour change techniques utilising the technical features of mobile devices and test the active ingredients of the health messages. ${ }^{61}$

\section{Conclusion}

Sustainable and wide reaching interventions promoting a healthy lifestyle in the antenatal period are urgently required to address negative maternal and child health outcomes. This study provides unique data demonstrating the feasibility of an mHealth intervention promoting healthy diet, physical activity and GWG recruiting in primary care, which has the ability to be scaled for wider dissemination. Significant intervention efficacy differences in GWG and physical activity suggest potential effects similar to other programs delivered via face-to-face, group and weight monitoring interventions.

\section{Disclosure of interests}

None declared. The ICMJE disclosure forms are available as online supporting information.

\section{Contribution to authorship}

JCW conceived and designed the study, composed the content and implementation procedures, project managed the implementation, analysed the results and drafted the manuscript. JCW and KJC secured funding. BF designed the texting software and contributed to the texting strategy and text message library. ML oversaw the study recruitment. SAW and EAMc contributed to the intervention content. JCW, SAW, ML, KB, DC, EAMc, BF, RW, RM and KJC contributed to the study design and procedures and read and approved the final manuscript. 


\section{Details of ethics approval}

The trial was approved by the Deakin University (2014-026: 20/2/2014)) and Mercy Hospital for Women (R13-64: 30/1/2014) Human Research Ethics Committees.

\section{Funding}

This study is supported from funding from the Lord Mayors Charitable Foundation, Melbourne, Australia. JW is supported by a Sidney Myer Health Scholarship, SW by a Queensland Health - Health Research Fellowship and KB by a National Health and Medical Research Council (NHMRC) Principal Research Fellowship (ID 1042442). The contents of this manuscript are the responsibility of the authors and do not reflect the views of the funding bodies.

\section{Acknowledgements}

The authors acknowledge the contribution of the women participating in the txt4two study. The authors also acknowledge the assistance of the following researchers and health professionals: Anne Griffiths (Deakin University); Alexis Shub (University of Melbourne); and Deborah Pidd, Elise Fraser, Gabrielle Pell and Nelly Moshonas (Mercy Hospital for Women)

\section{Supporting information}

Additional supporting information may be found in the online version of this article:

Table S1. Intervention components

Table S2. Mapping of the intervention

Table S3. Process Evaluation

Table S4. Perceived helpfulness of program and constituent behaviour change elements

Table S5. Self-reported physical activity: between group comparisons of intervention effect

Table S6. Self-reported diet: between group comparisons of intervention effect 


\section{References}

1. Zeisel SH. Epigenetic mechanisms for nutrition determinants of later health outcomes. Am J Clin Nutr 2009;89(5):1488S-93S.

2. Gluckman P, Hanson M. Developmental and epigenetic pathways to obesity: an evolutionary-developmental perspective. Int J Obes 2008;32:S62-S71.

3. Faucher MA, Hastings-Tolsma M, Song JJ, Willoughby DS, Bader SG. Gestational weight gain and preterm birth in obese women: a systematic review and meta-analysis. BJOG 2016;123(2):199206.

4. Haugen M, Brantsaeter AL, Winkvist A, Lissner L, Alexander J, Oftedal B, et al. Associations of pre-pregnancy body mass index and gestational weight gain with pregnancy outcome and postpartum weight retention: a prospective observational cohort study. BMC Preg Child 2014;14(1):201.

5. Laitinen J, Jaaskelainen A, Hartikainen AL, Sovio U, Vaarasmaki M, Pouta A, et al. Maternal weight gain during the first half of pregnancy and offspring obesity at 16 years: a prospective cohort study. BJOG 2012;119(6):716-23.

6. Saldana TM, Siega-Riz AM, Adair LS, Suchindran C. The relationship between pregnancy weight gain and glucose tolerance status among black and white women in central North Carolina. Am J Obstet Gynecol 2006;195(6):1629-35.

7. Boyle A, Timofeev J, Halscott T, Desale S, Driggers RW, Ramsey PS. Is 40 the new 30?: pregnancy outcomes by degree of weight gain among obesity subclasses. Obstet Gynecol 2014;123 Suppl 1:41S.

8. Mamun AA, Callaway LK, O'Callaghan MJ, Williams GM, Najman JM, Alati R, et al. Associations of maternal pre-pregnancy obesity and excess pregnancy weight gains with adverse pregnancy outcomes and length of hospital stay. BMC Preg Child 2011;11:62.

9. Kowal C, Kuk J, Tamim H. Characteristics of weight gain in pregnancy among Canadian women. Matern Child HIth J 2012;16(3):668-76.

10. IOM, NRC, Rasmussen $\mathrm{K}$, Yaktine $\mathrm{A}$. Weight gain during pregnancy: reexamining the guidelines. Washington: The National Academies Press; 2009.

11. Weisman CS, Hillemeier MM, Downs DS, Chuang CH, Dyer A-M. Preconception predictors of weight gain during pregnancy: prospective findings from the Central Pennsylvania Women's Health Study. Women Health Iss 2010;20(2):126-32.

This article is protected by copyright. All rights reserved 
12. Muktabhant B, Lawrie TA, Lumbiganon P, Laopaiboon M. Diet or exercise, or both, for preventing excessive weight gain in pregnancy. The Cochrane Database Of Systematic Reviews 2015;6:CD007145.

13. Glasgow RE, Emmons KM. How can we increase translation of research into practice? Types of evidence needed. Annu Rev Public Health 2007;28:413-33.

14. Olander EK, Atkinson L. Obese women's reasons for not attending a weight management service during pregnancy. Acta Obstet Gynecol Scand 2013;92(10):1227-30.

15. World Health Organization. mHealth: New horizons for health through mobile technologies. Geneva; 2011.

16. Free C, Phillips G, Galli L, Watson L, Felix L, Edwards P, et al. The effectiveness of mobilehealth technology-based health behaviour change or disease management interventions for health care consumers: A systematic review. PLoS Med 2013;10(1):1-45.

17. O'Brien OA, McCarthy M, Gibney ER, McAuliffe FM. Technology-supported dietary and lifestyle interventions in healthy pregnant women: a systematic review. Eur J Clin Nutr 2014;68(7):760-66.

18. McCarthy EA, Walker SP, Ugoni A, Lappas M, Leong O, Shub A. Self-weighing and simple dietary advice for overweight and obese pregnant women to reduce obstetric complications without impact on quality of life: a randomised controlled trial. BJOG 2016;123(6):965-73.

19. Soltani H, Duxbury AMS, Arden MA, Dearden A, Furness PJ, Garland C. Maternal obesity management using mobile technology: A feasibility study to evaluate a text messaging based complex intervention during pregnancy. J Obes 2015;2015:10.

20. Pollak KI, Alexander SC, Bennett G, Lyna P, Coffman CJ, Bilheimer A, et al. Weight-related SMS texts promoting appropriate pregnancy weight gain: A pilot study. Patient Educ Couns 2014;97(2):256-60.

21. Herring SJ, Cruice JF, Bennett GG, Rose MZ, Davey A, Foster GD. Preventing excessive gestational weight gain among African American women: A randomized clinical trial. Obesity 2016;24(1):30-6.

22. Willcox JC, Campbell KJ, McCarthy EA, Wilkinson SA, Lappas M, Ball K, et al. Testing the feasibility of a mobile technology intervention promoting healthy gestational weight gain in pregnant women (txt4two) - study protocol for a randomised controlled trial. Trials 2015;16(1):209-. 23. Willcox JC, Campbell KJ, McCarthy EA, Lappas M, Ball K, Crawford D, et al. Gestational weight gain information: seeking and sources among pregnant women. BMC Pregnancy Childbirth 2015;15(1):1-10.

This article is protected by copyright. All rights reserved 
24. Willcox JC, van der Pligt P, Ball K, Wilkinson SA, Lappas M, McCarthy EA, et al. Views of women and health professionals on mHealth lifestyle interventions in pregnancy: A qualitative investigation. JMIR mHealth and uHealth 2015;3(4):e99.

25. Chan A-W, Tetzlaff JM, Altman DG, Laupacis A, Gøtzsche PC, Krleža-Jerić K, et al. SPIRIT 2013 statement: defining standard protocol items for clinical trials. Ann Intern Med 2013;158(3):200-7.

26. Eysenbach G. CONSORT-EHEALTH: improving and standardizing evaluation reports of Webbased and mobile health interventions. J Med Internet Res 2011;13(4):e126-e.

27. Eysenbach G. CONSORT-EHEALTH: Implementation of a Checklist for Authors and Editors to Improve Reporting of Web-Based and Mobile Randomized Controlled Trials. Stud Health Technol Inform 2013;192:657-61.

28. Teare MD, Dimairo M, Shephard N, Hayman A, Whitehead A, Walters SJ. Sample size requirements to estimate key design parameters from external pilot randomised controlled trials: a simulation study. Trials 2014;15(1):264-.

29. National Health and Medical Research Council. Australian Dietary Guidelines. Canberra; 2013.

30. Australian Government. Australia's Physical Activity \& Sedentary Behaviour Guidelines for Adults. Canberra; 2014.

31. Bandara A. Social foundations of thought and action: A social cognitive theory. Englewood Cliffs: Prentice-Hall, Inc.; 1986.

32. Michie S, Ashford S, Sniehotta FF, Dombrowski SU, Bishop A, French DP. A refined taxonomy of behaviour change techniques to help people change their physical activity and healthy eating behaviours: The CALO-RE taxonomy. Psychol Health 2011;26(11):1479-98.

33. Fjeldsoe BS, Miller YD, O'Brien JL, Marshall AL. Iterative development of MobileMums: a physical activity intervention for women with young children. Int J Behav Nutr Phys Act 2012;9(151):1.

34. Free C, Knight R, Robertson S, Whittaker R, Edwards P, Zhou W, et al. Smoking cessation support delivered via mobile phone text messaging (txt2stop): a single-blind, randomised trial. Lancet 2011;378(9785):49-55.

35. Saunders RP, Evans MH, Joshi P. Developing a process-evaluation plan for assessing health promotion program implementation: A how-to guide. Health promotion practice 2005 April 1, 2005;6(2):134-47.

36. Ball K, Abbott G, Cleland V, Timperio A, Thornton L, Mishra G, et al. Resilience to obesity among socioeconomically disadvantaged women: the READI study. Int J Obes 2012;36(6):855-65.

This article is protected by copyright. All rights reserved 
37. McLennan W, Podger AS. National Nutrition Survey Users' Guide, 1995: Australian Bureau of Statistics [and] Commonwealth Department of Health and Family Services; 1998.

38. Marks GC, Webb K, Rutishauser IH, Riley M. Monitoring food habits in the Australian population using short questions. Canberra: Commonwealth of Australia 2001;200.

39. Sanigorski AM, Bell AC, Swinburn BA. Association of key foods and beverages with obesity in Australian schoolchildren. Public Health Nutr 2007;10(02):152-7.

40. Smith KJ, McNaughton SA, Cleland VJ, Crawford D, Ball K. Health, behavioral, cognitive, and social correlates of breakfast skipping among women living in socioeconomically disadvantaged neighborhoods. The Journal of nutrition 2013:jn. 113.181396.

41. Chasan-Taber L, Schmidt MD, Roberts DE, Hosmer D, Markenson G, Freedson PS.

Development and validation of a pregnancy physical activity questionnaire. Med Sci Sports Exerc 2004 Oct;36(10):1750-60.

42. Evenson KR, Chasan-Taber L, Symons Downs D, Pearce EE. Review of Self-reported Physical Activity Assessments for Pregnancy: Summary of the Evidence for Validity and Reliability. Paediatr Perinat Epidemiol 2012;26(5):479-94.

43. Campbell MK, Piaggio G, Elbourne DR, Altman DG. Consort 2010 statement: extension to cluster randomised trials. BMJ 2012 2012-09-04 11:03:48;345.

44. Whittaker R, Merry S, Dorey E, Maddison R. A development and evaluation process for mHealth interventions: Examples from New Zealand. J Health Comm 2012;17:11-21.

45. Bowen DJ, Kreuter M, Spring B, Cofta-Woerpel L, Linnan L, Weiner D, et al. How we design feasibility studies. Am J Prev Med 2009;36(5):452-7.

46. Hill B, Skouteris H, Fuller-Tyszkiewicz M. Interventions designed to limit gestational weight gain: a systematic review of theory and meta-analysis of intervention components. Obes Rev 2013;14(6):435-50.

47. Michie S, Richardson M, Johnston M, Abraham C, Francis J, Hardeman W, et al. The Behavior Change Technique Taxonomy (v1) of 93 Hierarchically Clustered Techniques: Building an International Consensus for the Reporting of Behavior Change Interventions. Ann Behav Med 2013;46(1):81-95.

48. Kristal AR, Peters U, Potter JD. Is it time to abandon the food frequency questionnaire? Cancer Epidemiology Biomarkers \& Prevention 2005;14(12):2826-8.

49. SchrÖDer H, Covas MI, Marrugat J, Vila J, Pena A, AlcÁNtara M, et al. Use of a three-day estimated food record, a 72-hour recall and a food-frequency questionnaire for dietary assessment in a Mediterranean Spanish population. Clin Nutr 2001;20(5):429-37.

This article is protected by copyright. All rights reserved 
50. Mhurchu CN, Whittaker R, McRobbie H, Ball K, Crawford D, Michie J, et al. Feasibility, acceptability and potential effectiveness of a mobile health (mHealth) weight management programme for New Zealand adults. BMC obesity 2014;1(1):10.

51. Thangaratinam S, Rogozińska E, Jolly K, Glinkowski S, Roseboom T, Tomlinson JW, et al. Effects of interventions in pregnancy on maternal weight and obstetric outcomes: meta-analysis of randomised evidence. BMJ 2012 2012-05-17 23:32:30;344:e2088.

52. Guerriero C, Cairns J, Roberts I, Rodgers A, Whittaker R, Free C. The cost-effectiveness of smoking cessation support delivered by mobile phone text messaging: Txt2stop. European Journal of Health Economics 2013;14(5):789-97.

53. Shirazian T, Monteith S, Friedman F, Rebarber A. Lifestyle modification program decreases pregnancy weight gain in obese women. Am J Perinatol 2010;27(5):411-4.

54. Vinter CA, Jensen DM, Ovesen P, Beck-Nielsen H, Jørgensen JS. The LiP (Lifestyle in Pregnancy) study: a randomized controlled trial of lifestyle intervention in 360 obese pregnant women. Diabetes Care 2011;34(12):2502-7.

55. Brown MJ, Sinclair M, Liddle D, Hill AJ, Madden E, Stockdale J. A systematic review investigating healthy lifestyle interventions incorporating goal setting strategies for preventing excess gestational weight gain. PLoS One 2012;7(7):5.

56. Kim HK, Niederdeppe J, Graham M, Olson C, Gay G. Effects of online self-regulation activities on physical activity among pregnant and early postpartum women. Journal of health communication $2015 / / ; 20(10): 1115-24$.

57. Sui Z, Turnbull D, Dodd J. Enablers of and barriers to making healthy change during pregnancy in overweight and obese women. Australasian Medical Journal 2013;6(11):565-77.

58. Chuang $\mathrm{CH}$, Stengel MR, Hwang SW, Velott D, Kjerulff KH, Kraschnewski JL. Behaviours of overweight and obese women during pregnancy who achieve and exceed recommended gestational weight gain. Obes Res Clin Pract 2014;8(6):e577-e83.

59. Baker CW, Carter AS, Cohen LR, Brownell KD. Eating attitudes and behaviors in pregnancy and postpartum: global stability versus specific transitions. Ann Behav Med 1999;21(2):143-8.

60. Miles S, Scaife V. Optimistic bias and food. Nutrition Research Reviews 2003;16(1):3-19.

61. Huberty J, Rowedder L, Hekler E, Adams M, Hanigan E, McClain D, et al. Development and design of an intervention to improve physical activity in pregnant women using Text4baby. Trans/ Behav Med 2016;6(2):285-94.

This article is protected by copyright. All rights reserved 
Table 1: Baseline characteristics of participants in the txt4two study

\begin{tabular}{|c|c|c|c|}
\hline & $\begin{array}{l}\text { Overall } \\
(n=91)\end{array}$ & $\begin{array}{l}\text { Control } \\
(n=46)\end{array}$ & $\begin{array}{c}\text { Intervention } \\
\qquad(n=45)\end{array}$ \\
\hline \multicolumn{4}{|l|}{ Socioeconomic } \\
\hline \multicolumn{2}{|l|}{ Birth country, n (\%) } & $32.0 \pm 5.1$ & $33.0 \pm 3.4$ \\
\hline Australia & $70(77)$ & $34(74)$ & $36(80)$ \\
\hline Other & $21(23)$ & $12(26)$ & $9(20)$ \\
\hline \multicolumn{4}{|l|}{ Language spoken at home, n (\%) } \\
\hline English & $72(79)$ & $35(76)$ & $37(82)$ \\
\hline Other & $19(21)$ & $11(24)$ & $8(18)$ \\
\hline \multicolumn{4}{|l|}{ Education, n (\%) } \\
\hline Secondary education or less & $19(21)$ & $11(24)$ & $8(18)$ \\
\hline Trade/certificate & $33(36)$ & $14(30)$ & $19(42)$ \\
\hline University & $39(43)$ & $21(46)$ & $18(40)$ \\
\hline \multicolumn{4}{|l|}{ Relationship status, n (\%) } \\
\hline Married/De facto & $88(97)$ & $45(98)$ & $43(96)$ \\
\hline Separated/widowed/ single & $3(3)$ & $1(2)$ & $2(4)$ \\
\hline \multicolumn{4}{|l|}{ Household income, $\mathrm{n}(\%)^{\mathbf{a}}$} \\
\hline$<\$ 51,999$ & $16(18)$ & $11(24)$ & $8(18)$ \\
\hline$\$ 52-77,999$ & $11(12)$ & $3(7)$ & $15(34)$ \\
\hline$\$ 78-99,9$ & $28(31)$ & $13(29)$ & $15(34)$ \\
\hline$>\$ 99,99$ & $27(30)$ & $12(27)$ & $1(2)$ \\
\hline Undisclosed & $7(8)$ & $6(13)$ & $8(18)$ \\
\hline \multicolumn{4}{|l|}{ Pregnancy } \\
\hline Weeks' gestation, mean \pm SD & $15.5 \pm 2.0$ & $15.8 \pm 1.7$ & $15.2 \pm 2.3$ \\
\hline \multicolumn{4}{|l|}{ Parity, n (\%) } \\
\hline Nulliparous & $42(46)$ & $20(43)$ & $22(49)$ \\
\hline Parity $\geq 1$ & $49(54)$ & $26(57)$ & $23(51)$ \\
\hline $\begin{array}{l}\text { Pre-pregnancy BMI }\left(\mathrm{kg} / \mathrm{m}^{2}\right) \text {, mean } \\
\pm \mathrm{SD}\end{array}$ & $31.0 \pm 5.1$ & $29.6 \pm 3.8$ & $32.5 \pm 5.8$ \\
\hline
\end{tabular}

BMI, body mass index; SD, standard deviation

${ }^{\mathrm{a}} \mathrm{A} \$$, Australian dollar

This article is protected by copyright. All rights reserved 


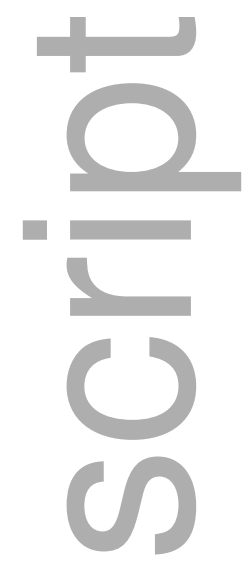

Table 2: Maternal GWG: between group comparisons of intervention effect ${ }^{\mathrm{a}}$

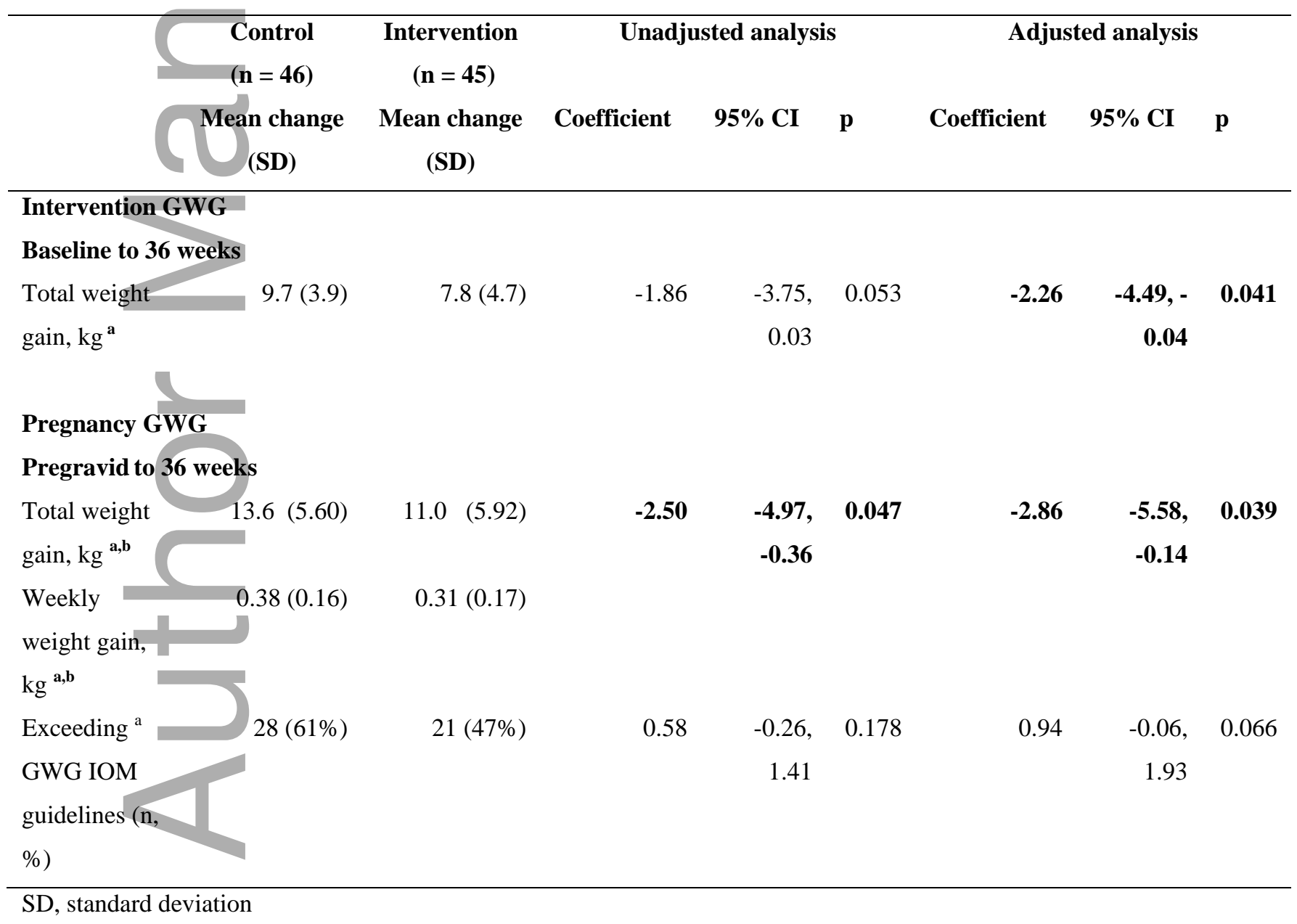

${ }^{a}$ Adjusted for pre-pregnancy or study entry weight, age, education, ppBMI, parity, gestational diabetes, and number of days in the study.

This article is protected by copyright. All rights reserved 
${ }^{\mathrm{b}}$ Self-reported pre-pregnancy weight

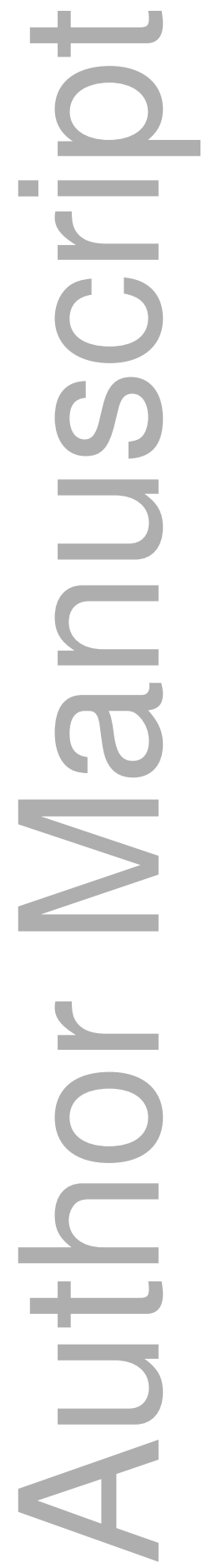

This article is protected by copyright. All rights reserved 


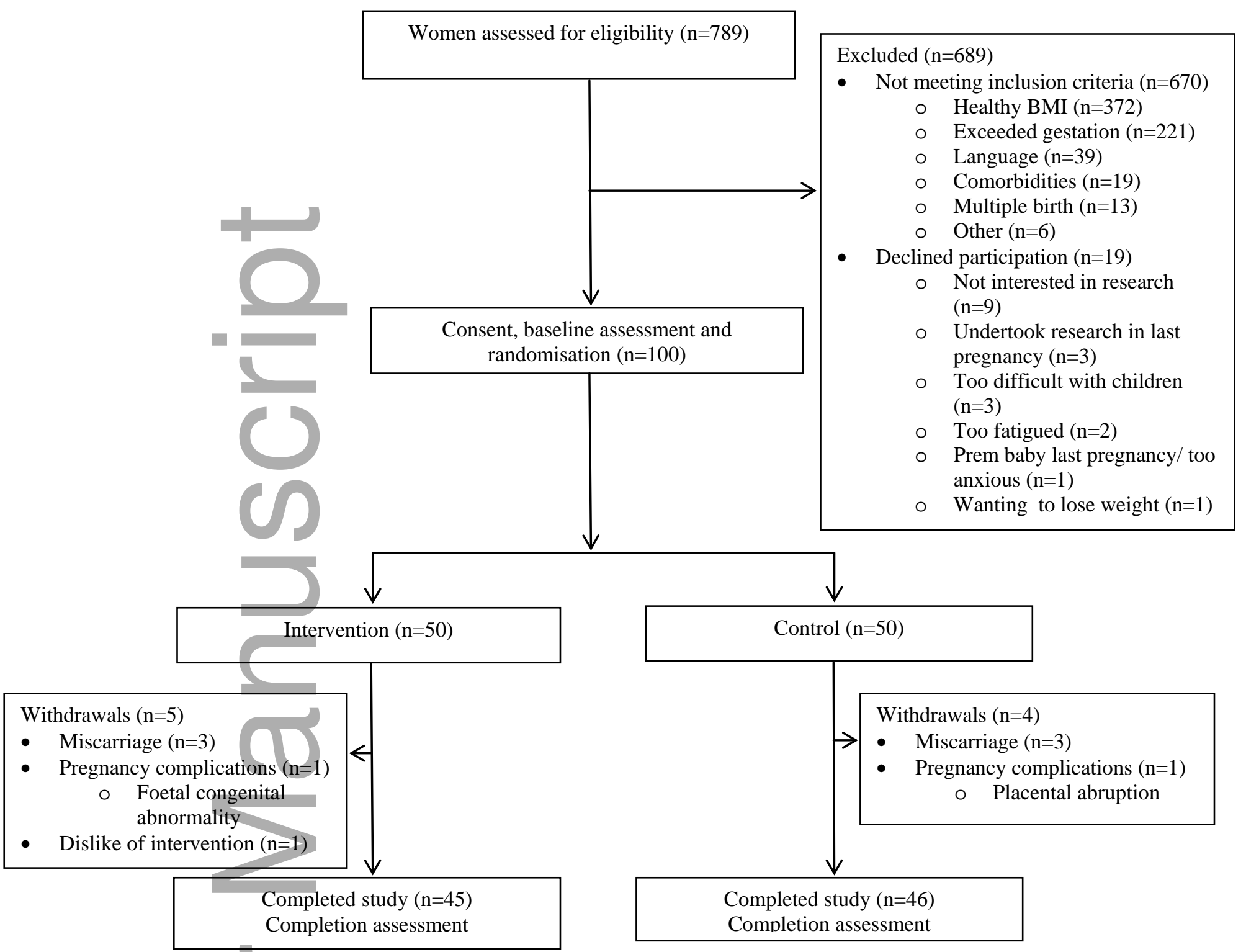

Figure 1: Enrolment of participants in the txt4two study 


\section{University Library}

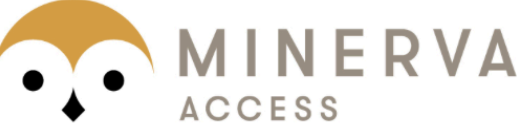

A gateway to Melbourne's research publications

Minerva Access is the Institutional Repository of The University of Melbourne

Author/s:

Willcox, JC;Wilkinson, SA;Lappas, M;Ball, K;Crawford, D;McCarthy, EA;Fjeldsoe,

$\mathrm{B} ;$ Whittaker, R;Maddison, R;Campbell, KJ

Title:

A mobile health intervention promoting healthy gestational weight gain for women entering pregnancy at a high body mass index: the txt4two pilot randomised controlled trial

Date:

2017-10-01

Citation:

Willcox, J. C., Wilkinson, S. A., Lappas, M., Ball, K., Crawford, D., McCarthy, E. A., Fjeldsoe, B., Whittaker, R., Maddison, R. \& Campbell, K. J. (2017). A mobile health intervention promoting healthy gestational weight gain for women entering pregnancy at a high body mass index: the txt4two pilot randomised controlled trial. BJOG-AN INTERNATIONAL JOURNAL OF OBSTETRICS AND GYNAECOLOGY, 124 (11), pp.1718-1728. https:// doi.org/10.1111/1471-0528.14552.

Persistent Link:

http://hdl.handle.net/11343/292459 